Signal \& Image Processing : An International Journal (SIPIJ) Vol.6, No.5, October 2015

\title{
COLOR IMAGE SEGMENTATION USING AN EFFICIENT FUZZY BASED WATERSHED APPROACH
}

\author{
Dibya Jyoti Bora, Anil Kumar Gupta and Fayaz Ahmad Khan \\ Department of Computer Science And Applications, \\ Barkatullah University, Bhopal(M.P.), India
}

\begin{abstract}
Color image segmentation is a very emerging topic in current image processing research. An optimal technique for the same is always sought by the researchers of this field. In this paper, an efficient approach for color image segmentation is proposed. Here, the input color image is first converted from RGB to HSV color space. The V channel of the HSV converted image is extracted and normalized between 0 and 1 . Then this normalized $V$ channel is sent as input to Fuzzy C Means (FCM) algorithm. The fuzzy segmented image is then thresholded with Otsu's method. The thresholded image is then filtered by sobel filter and sent as input to the Meyer's watershed algorithm. This produces the final segmented image of the original color image. The proposed approach is found very efficient after analyzing and comparing the results with previously existed watershed algorithm in terms of the MSE and PSNR values.
\end{abstract}

\section{KEYWORDS}

Color Image Segmentation, FCM, Image Normalization, Otsu's Method, Sobel Filter, Watershed Algorithm

\section{INTRODUCTION}

Image segmentation is the process of partitioning an image into uniform and non overlapping regions so that meaningful information can be extracted from the segmented image [1]. These regions are known as segments. The union of any two neighboring segments yields a heterogeneous segment. Homogeneity of the segmented regions is determined with respect to the factors like color, texture, motion etc. Image segmentation results a set of segments that collectively cover the entire image, or a set of contours extracted from the image [2]. Since image may be gray scale or color, so image segmentation may be either gray scale or color depending on the type of the image. But color image segmentation has sometimes offered more benefits than gray scale segmentation because our human eyes are more adjustable to brightness, so, can identify thousands of color at any point of a complex image, while only a dozens of gray scale are possible to be identified at the same time[3]. Image segmentation methods can be broadly classified into seven groups [4]: (1) Histogram thresholding, (2) Clustering (Soft and Hard), (3) Region growing, region splitting and merging, (4) Edge-based, (5) Physical model based, (6) Fuzzy approaches, and (7) Neural network and GA (Genetic algorithm) based approaches. In this 
Signal \& Image Processing : An International Journal (SIPIJ) Vol.6, No.5, October 2015

paper, we attempt to combine the powers of fuzzy clustering with region growing method. There exists a number of fuzzy clustering methods for image segmentation [5], but we choose FCM(Fuzzy C Means) for our proposed approach. Mayer's watershed algorithm is one of the famous region growing methods for image segmentation task. We have chosen this classical algorithm for our approach. But watershed algorithm suffers from over segmentation problem. So, a proper preprocessing is required before applying this algorithm. FCM, Otsu's Thresolding Method and Sobel filter perform this task of preprocessing. Also, choosing a proper color space is utmost necessary for color image segmentation [6]. We have chosen HSV for our task. First, RGB image is converted to HSV one and V channel is extracted from the HSV image. The V channel is normalized between 0 and 1 . The normalized image is then sent as input to the FCM algorithm. The output image of the FCM algorithm is thresholded with Otsus's global thresholding method. The thresholded image is then filtered with Sobel filter. Finally, this filtered image is segmented with Watershed method and thereby we get the final segmented image of our input color image.

The paper is organized as follows: Section 2 gives the review of literature in this field. In Section 3, flowchart of the proposed method can be found. Then, the topics concerned with the proposed approach: HSV Color Space, Image Normalization, FCM, Otsu's Thresholding, Sobel Filter and Watershed Algorithm are discussed in the sections 4, 5, 6, 7, 8 and 9 respectively. Experiments and results are presented in the Section 10. Finally, in the Section 11, conclusion and future enhancement is given.

\section{REVIEW OF LITERATURE}

In [7], the author proposed an image segmentation method that showed the use of fuzzy c-means clustering in image segmentation. This method is based on a basic region growing method and uses membership grades' of pixels to classify pixels into appropriate segments. Images were in RGB color space, as feature space was used $\mathrm{L}^{*} \mathrm{u}^{*} \mathrm{v}^{*}$ color space. Results of the experiments showed that the method is very efficient in dealing with color image segmentation but one of the demands was not reached that was the demand of simplifying borders.

In [8], the authors proposed a new fuzzy c means algorithm that relies on a new efficient cluster center initialization and color quantization allowing faster and more accurate convergence such that it is suitable to segment very large color images. The experiments on real images proved the efficiency of the algorithm in terms of both accuracy and computation time.

In [9], the authors proposed an approach where the segmentation of various color spaces such as RGB,HSV, YIQ and XYZ are combined to give more accurate segmentation result compared to segmentation of single color space. K-Means and Effective robust kernelized fuzzy cmeans(ERKFCM) are used to segment the images. MSE and PSNR are used to evaluate the performance.

In [10], the authors proposed a color image segmentation method based on the combination of watershed algorithm with seed region growing algorithm. Here, watershed method is used to initialize the segmentation.

In [11], the authors presented an improved watershed image segmentation method, where, firstly, the morphological opening/closing reconstruction filter is applied to remove the image noise and 
Signal \& Image Processing : An International Journal (SIPIJ) Vol.6, No.5, October 2015

secondly, multi-scale structure elements are used to calculate morphological gradient. Also, the morphological gradient is modified by viscous morphological operators to remove the most irregular minimums. The region merging method based on neighbor regions edge value is applied after standard watershed transform to improve the segmentation result. Experiments showed that the method is effectively avoiding the over segmentation problem of watershed and also preserves the positions of regional contours.

In [12], a new approach for clustering based color image segmentation is proposed where KMeans algorithm is integrated with watershed algorithm. Also cosine distance measure is used in $\mathrm{K}-\mathrm{Means}$ algorithm. The color space chosen is HSV. A post processing with median filter is done to remove the noises that might come during the segmentation process. The approach is found to produce satisfactory segmentation results.

In [13], the authors proposed an improved fast watershed algorithm. The algorithm is fast in the sense that it scans the image only twice, one for generating connected components and other for labelling the catchment basins. The experimental results show that proposed algorithm produces expected results twice faster than existing algorithm.

In [14], a modified version of watershed algorithm is presented where an adaptive masking and a threshold mechanism are used over each color channel to overcome the over segmentation problem of watershed algorithm, before combining the segmentation from each channel to the final one. The approach is enhancing the segmentation result and also result is found more accurate as per the obtained values of image quality assessment metrics such as PSNR, MSE and Color Image Quality Measure (CQM) based on reversible YUV color transformation.

In [15], the authors presented a new method for image segmentation based on watershed transformation using mathematical morphology. To avoid over segmentation, the topological gradient method is adopted. The results obtained are found very efficient.

\section{FLOWCHART OF THE PROPOSED APPROACH}

The flowchart of the proposed approach is shown below:

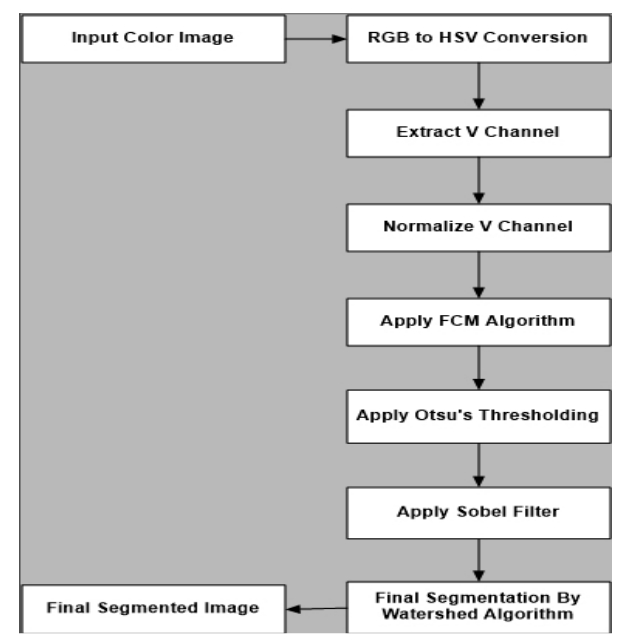

Figure 1.Flowchart of the Proposed Approach 
Signal \& Image Processing : An International Journal (SIPIJ) Vol.6, No.5, October 2015

\section{HSV COLOR SPACE}

In color image processing research, HSV is a frequently chosen color space [6]. This color space can be represented by a hexacone in three dimensions with a central vertical axis that represents the intensity. A diagrammatic view of the HSV Color space can be found in [16]:

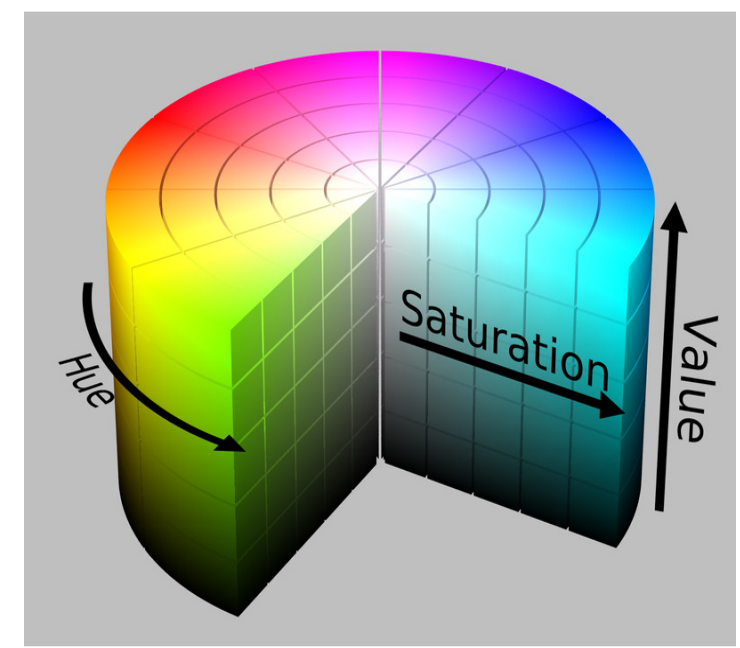

Figure 2.HSV Color Space

In HSV, 'H' stands for 'Hue'. 'Hue' is an angle in the range $[0,2 \pi]$ relative to the red axis with red at angle 0 , green at $2 \pi / 3$, blue at $4 \pi / 3$ and red again at $2 \pi$ [17]. 'S' stands for 'Saturation', which describes how pure the hue is with respect to a white reference. Saturation can be thought of as the depth or purity of color and is measured as a radial distance from the central axis with values between 0 at the center to 1 at the outer surface. When $S=0$ and one moves higher along the intensity axis then it goes from black to white through various shades of gray. Again, for a given intensity and hue, if the saturation is changed from 0 to 1 , the perceived color changes from a shade of gray to the most pure form of the color represented by its hue. ' $V$ ' stands for 'Value' which is a percentage that goes from 0 to 100. This range (from 0 to 100) can be thought as the amount of light illuminating a color. For example, when the hue is red and the value is high, the color looks bright. Again, if the value is low it looks dark [17].

\section{IMAGE NORMALIZATION}

By 'normalization', we usually mean a pre processing stage to find out a new range from an existing one stage [18]. In image processing, 'image normalization' is a process that changes the ranges of pixel intensity values [19]. Normalization of image is done in order to bring the image into a range that is more familiar or normal to the senses. This is some times also known as "contrast stretching" or "histogram stretching" [20]. Image normalization is always chosen for eliminating image variations (such as noise, illumination, or occlusion) that may arise during image acquisition and are totally not relevant to object identity, thereby trying to obtain a standard image with no artifacts [21]. In our proposed approach, the V channel of the HSV converted image is normalized in the range 0 and 1 using the formula:

Normalized $(\mathrm{V})=\{\mathrm{V}-\min (\mathrm{V})\} /\{\max (\mathrm{V})-\min (\mathrm{V})\}$; 
Signal \& Image Processing : An International Journal (SIPIJ) Vol.6, No.5, October 2015

Here $\mathrm{V}$ denotes the $\mathrm{V}$ channel, $\min (\mathrm{V})$ and $\max (\mathrm{V})$ denotes respectively the minimum and maximum values of $\mathrm{V}$, and Normalized $(\mathrm{V})$ is the normalized output image obtained after the mentioned normalization.

\section{FCM ALGORITHM}

FCM (Fuzzy C Means) is one of the famous data clustering technique wherein each data point belongs to a cluster to some degree that is specified by a membership grade [22]. One of the advantage of FCM is that it allows gradual memberships of data points to clusters measured as degrees in $[0,1]$ which in turn gives the flexibility to express that data points can belong to more than one cluster. Here, the algorithm iteratively tries to minimize the cost function:

$J_{m}=\sum_{i=1}^{N} \sum_{j=1}^{C} u_{1 \leq m<\infty}{ }^{m}\left\|x_{i}-c_{j}\right\|^{2}$

In the above equation, $\mathrm{m}$ is any real number greater than $1, u_{i j}$ is the degree of membership of pixel $\boldsymbol{x}_{i}$ in the cluster $j,\|*\|$ is any norm expressing the similarity between the concerned pixel and the center, the parameter $m$ controls the fuzziness of the partition[24]. Fuzzy partitioning is carried out through an iterative optimization of the objective function shown above, with the update of membership $u_{i j}$ and the cluster centers $c j$ by

$$
\begin{gathered}
u_{i j}=\frac{1}{\sum_{k=1}^{c}\left(\frac{\left\|x_{i}-c_{j}\right\|}{\left\|x_{i}-c_{k}\right\|}\right)^{\frac{2}{m-1}}} \\
c_{j}=\frac{\sum_{i=1}^{N}{u_{i j}}^{m} \bullet x_{i}}{\sum_{i=1}^{N} u_{i j}{ }^{m}}
\end{gathered}
$$

The iteration will stop if $\max _{i j}\left\{\left|u_{i j}{ }^{(k+1)}-u_{i j}{ }^{(k)}\right|\right\}<\in$ where $\in$ is a termination criteria between 0 and 1 , whereas $\mathrm{k}$ are the iteration steps. This procedure converges to a local minimum or a saddle point of $J_{m}$. We have chosen the FCM as an initial segmentation algorithm in our approach.

\section{OTSU'S THRESHOLDING METHOD}

Otsu Thresholding Method is a global thresholding technique [25]. This was proposed by Scholar Otsu in 1979. The method grants that an image to be thresholded consists of two groups of pixels or bi-model histrogram(foreground and background) and then evaluates the optimum threshold partitioning those two classes so that the intra-class variance is negligible. So, here we try to reduce the weighted sum of the variances of the two classes:

$\sigma_{\mathrm{w}}^{2}(\mathrm{t})=\omega 1(t) \sigma_{1}^{2}(t)+\omega 2(t) \sigma_{2}^{2}(t)$ 
Signal \& Image Processing : An International Journal (SIPIJ) Vol.6, No.5, October 2015

Where, $\omega \mathrm{i}$ are the probabilities of two class partitioned through a threshold $\mathrm{t}$ and $\sigma_{\mathrm{i}}^{2}$ are the discrepancies of these sets. Otsu proved that by minimizing the intra -class variances is same as maximizing the inter-class variance as:

$$
\begin{aligned}
\sigma^{2}(t)=\sigma^{2} & -\sigma_{\omega}^{2}(t) \\
& =\omega_{1}(t) \omega_{2}(t)\left[\mu_{1}(t)-\mu_{2}(t)\right]^{2}
\end{aligned}
$$

-which is stated in terms of the class probabilities $\mathrm{w}_{\mathrm{i}}$ and the class mean $\mu_{\mathrm{i}}$

The class probability $\mathrm{w}_{1}(\mathrm{t})$ will be calculated from the histogram as $\mathrm{t}$ :

$\mathrm{w}_{1}(\mathrm{t})=\sum_{0}^{t} P(i)$

and,

class mean, $\mu_{1}(\mathrm{t})=\sum_{0}^{t} P(i) X(i)$

where $X(i)$ refers to the value of the center of I in histogram bin. Similarly, $\mathrm{w}_{2}(\mathrm{t})$ and $\mu_{2}(\mathrm{t})$ can be calculated for the histograms of bins bigger than t. The class probabilities and the class mean can be calculated iteratively [25]. In our proposed,Otsu's method is chosen because of its capability towards better threshold selection for general real world noisy images with regard to uniformity and shape measures.

\section{SOBEL FILTER}

In edge detection algorithms, the Sobel filter (also, known as Sobel operator), named after Irwin Sobel[27], is generally used to create an image by emphasizing edges and transitions of the image. Sobel filter is a discrete differentiation operator which computes an approximation of the gradient of the image intensity function. This computation is based on convolving the image with a small, separable, and integer valued filter in horizontal and vertical direction and is therefore relatively inexpensive in terms of computations [28]. Sobel filter is an orthogonal gradient operator [29], where gradient corresponds to first derivative and gradient operator is a derivative operator. Two kernels: Gx and Gy are involved for every image ; where Gx is estimating the gradient in $\mathrm{x}$-direction while Gy estimating the gradient in y-direction. Then the absolute gradient magnitude will be given by:

$$
|\mathbf{G}|=\sqrt{ }\left(\mathbf{G} \mathbf{x}^{2}+\mathbf{G y}^{\mathbf{2}}\right)
$$

Although, this value is often approximated with [28][29] :

$$
|\mathbf{G}|=|\mathbf{G x}|+|\mathbf{G y}|
$$

We have chosen sobel operator because of its capacity of smoothing effect on the random noises of an image[24]. The edge elements, being differentially separated by two rows and columns on both sides, become enhanced which offer a very bright and thick look of the edges. 
Signal \& Image Processing : An International Journal (SIPIJ) Vol.6, No.5, October 2015

\section{WATERSHED TRANSFORMATION}

Watershed transformation is a powerful mathematical morphological tool for image segmentation task. The term "watershed", in geography, means the ridge that divides areas drained by different river systems. When an image is viewed as geological landscape, the watershed lines determine boundaries which separate image regions. The watershed transform computes catchment basins and ridgelines (also known as watershed lines), where catchment basins corresponding to image regions and ridgelines relating to region boundaries [30]. A diagrammatic view of watershed lines and catchment basins is [31]:

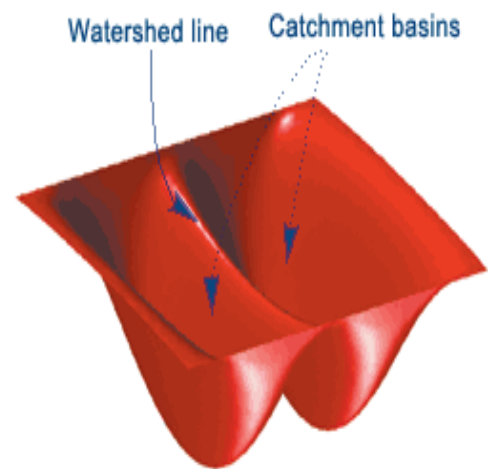

Figure 3.Watershed line and Catchment basins.

In our approach, we have chosen Meyer's watershed algorithm. The basic steps involved in this algorithm are [32]:

1. Add neighbors to priority queue, sorted by value.

2. Choose local minima as region seeds.

3. Take top priority pixel from queue

1. If all labeled neighbors have same label, assign to pixel

2. Add all non-marked neighbors

4. Repeat step 3 until finished.

\section{EXPERIMENTS AND RESULTS}

The proposed approach has been implemented in Matlab. Images for the experiments are collected from Matlab demo images [33] and Berkeley Image Segmentation dataset [34]. The performance is evaluated and compared in terms of two parameters MSE and PSNR whose brief introductions are given below:

The MSE (Mean Squared Error)is the cumulative squared error between the compressed and the original image, whereas PSNR(Peak Signal to Noise Ratio) is the peak error[35][36]. MSE can be computed using the following formula [36] is:

MSE $=\sum_{y=1}^{M} \sum_{x=1}^{N}\left[I(x, y)-I^{\prime}(x, y)\right]^{2}$ 
Signal \& Image Processing : An International Journal (SIPIJ) Vol.6, No.5, October 2015

where, $I(x, y)$ is the original image, $I^{\prime}(x, y)$ is its noisy approximated version (which is actually the decompressed image) and M,N are the dimensions of the images value for MSE implies lesser error.

The formula for PSNR[36] is:

$\mathrm{PSNR}=10 \log _{10}\left(M A X i^{2} / M S E\right)$

Where, MAXi is the maximum possible pixel value of the image. A higher value of PSNR is always preferred as it implies the ratio of Signal to Noise will be higher. 'signal' here is the original image, and the 'noise' is the error in reconstruction.

\section{Experiments for Image 1:}

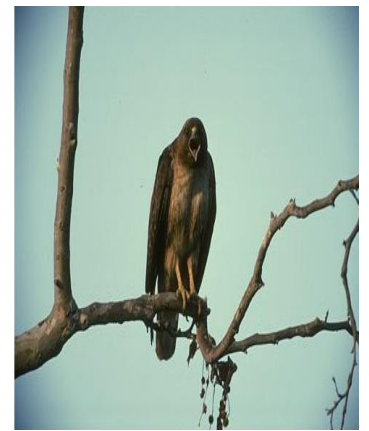

Figure 4(i).Original Image (Image 1)

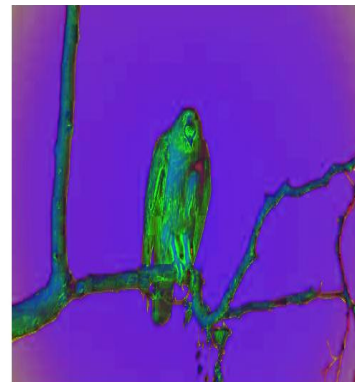

Figure 4(ii).HSV Converted Image

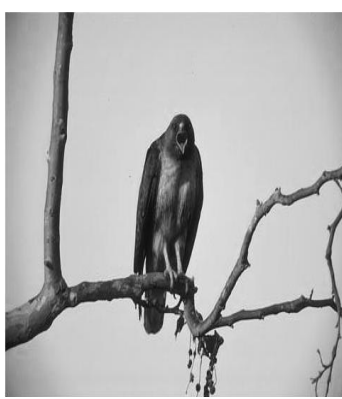

Figure 4(iii).V Channel 
Signal \& Image Processing : An International Journal (SIPIJ) Vol.6, No.5, October 2015

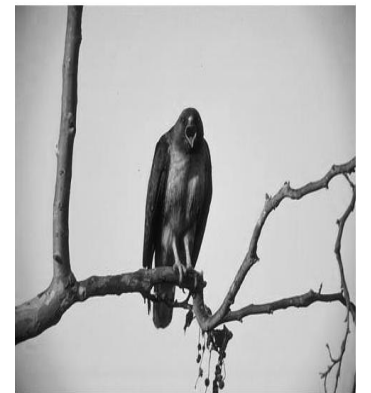

Figure 4(iv).Normalized V Channel

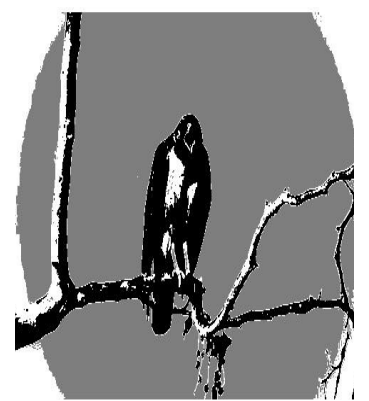

Figure 4(v).Initial Segmentation by FCM

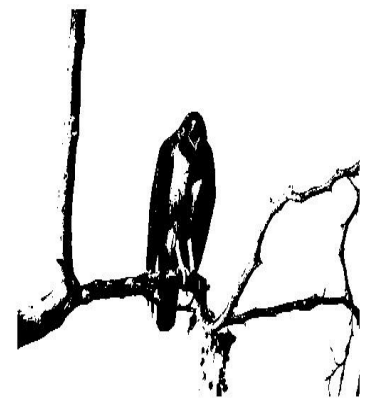

Figure 4(vi).After Applying Otsu's Thresholding

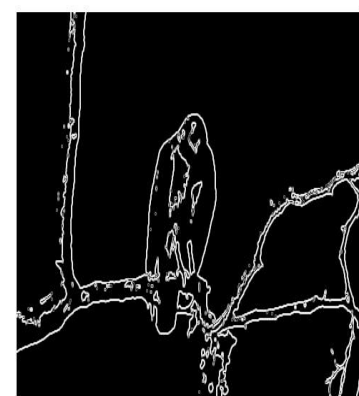

Figure 4(vii).After Applying Sobel Filter 
Signal \& Image Processing : An International Journal (SIPIJ) Vol.6, No.5, October 2015

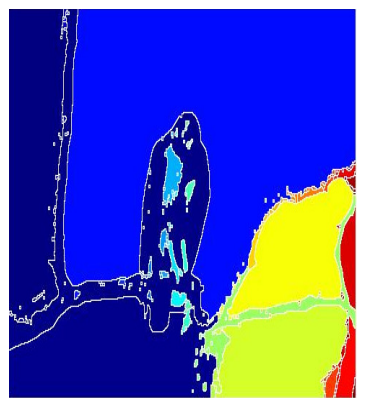

Figure 4(viii).Final Segmented Image after Applying Watershed Algorithm

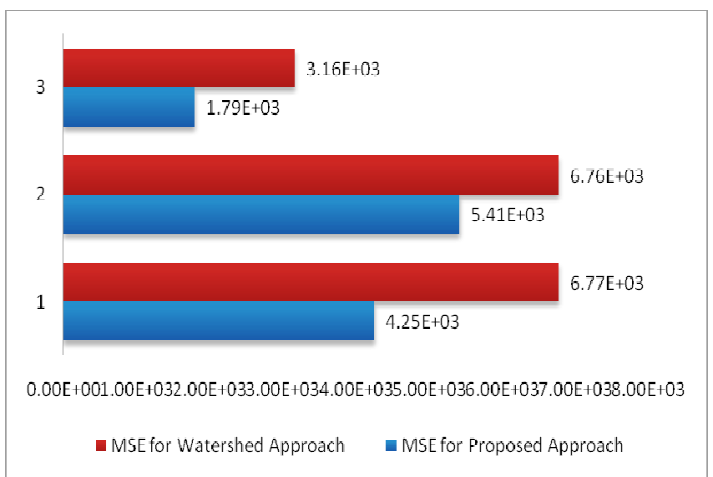

Figure 5(i).MSE Comparison for the Image 1

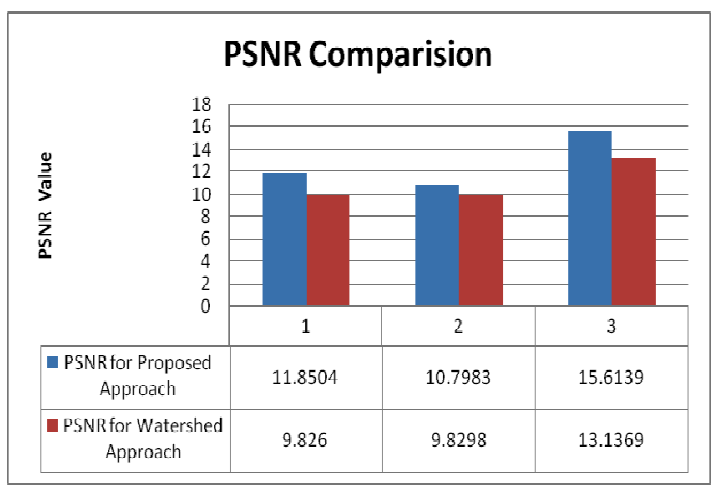

Figure 5(ii). PSNR Comparison for the Image 1

\section{Experiments for Image 2:}

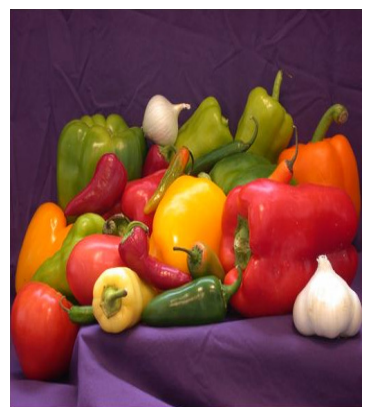

Figure 6(i). Original Image (Image 2) 
Signal \& Image Processing : An International Journal (SIPIJ) Vol.6, No.5, October 2015

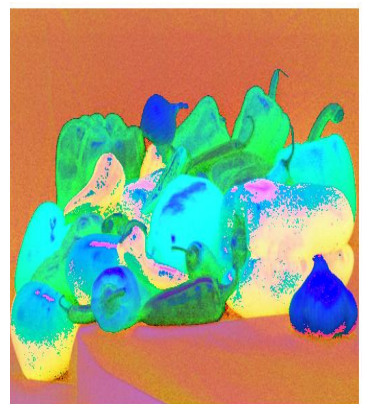

Figure 6(ii). HSV Converted Image

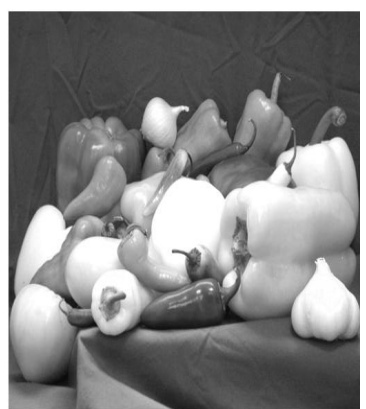

Figure 6(iii). V Channel

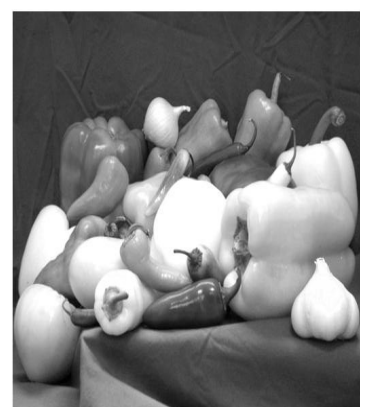

Figure 6(iv). Normalized V Channel

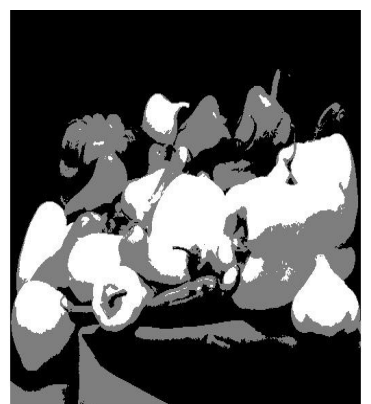

Figure 6(v). Initial Segmentation by FCM 
Signal \& Image Processing : An International Journal (SIPIJ) Vol.6, No.5, October 2015

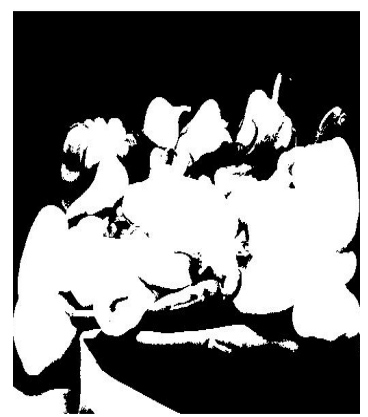

Figure 6(vi). After Applying Otsu's Thresholding

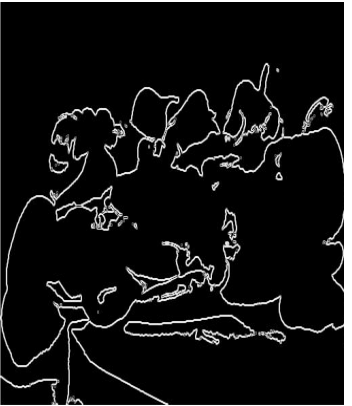

Figure 6(vii). After Applying Sobel Filter

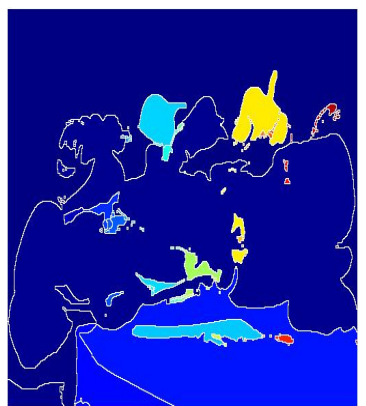

Figure 6(viii). Final Segmented Image after Applying Watershed Algorithm

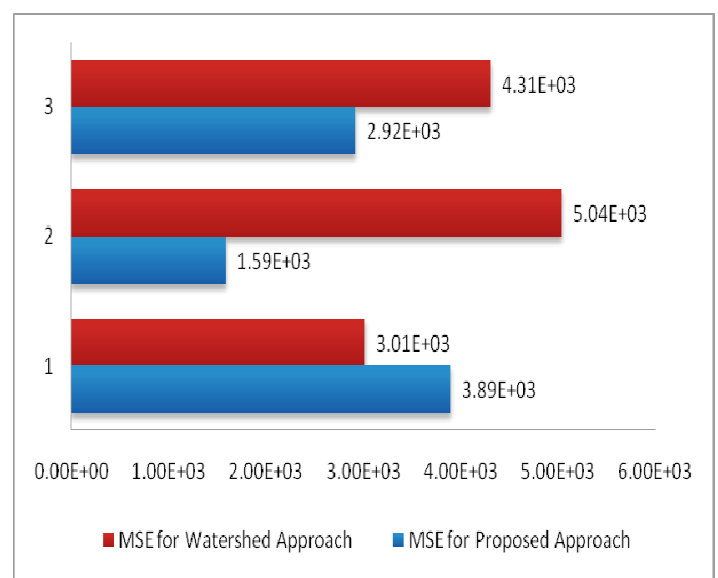

Figure 7(i).MSE Comparison for the Image 2. 
Signal \& Image Processing : An International Journal (SIPIJ) Vol.6, No.5, October 2015

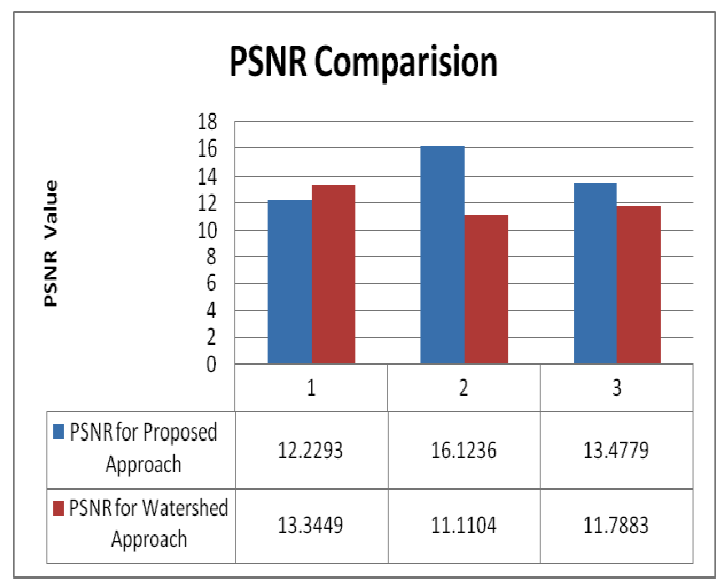

Figure 7(ii).PSNR Comparison for the Image 2

\section{Experiments for the Image 3:}

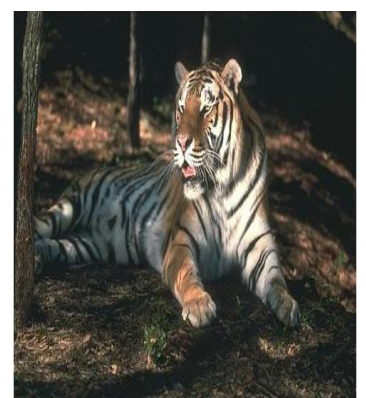

Figure 8(i).Original Image (Image 3)

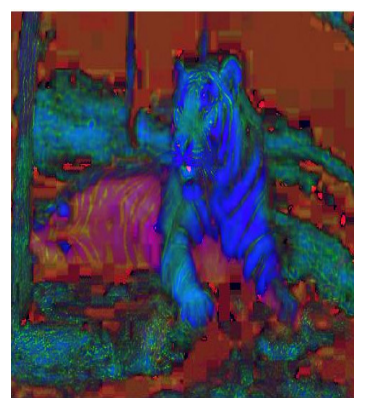

Figure 8(ii).HSV Converted Image

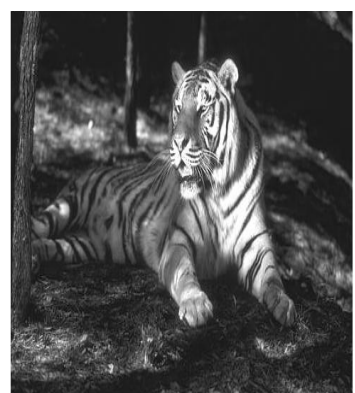

Figure 8(iii).V Channel 
Signal \& Image Processing : An International Journal (SIPIJ) Vol.6, No.5, October 2015

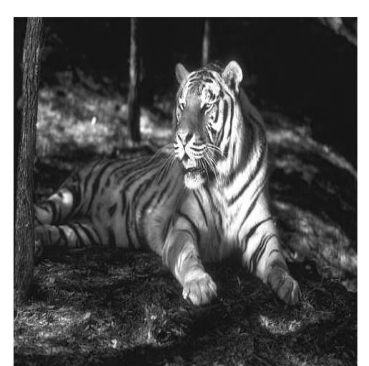

Figure 8(iv).Normalized V Channel

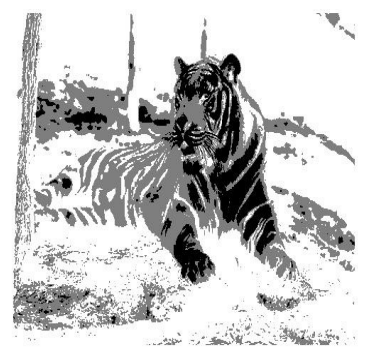

Figure $8(v)$. Initial Segmentation by FCM

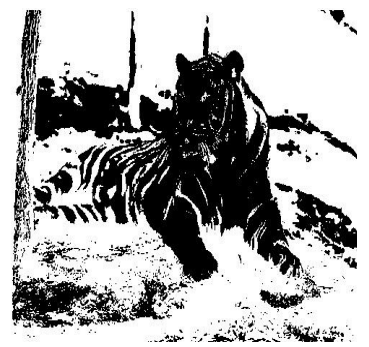

Figure 8(vi).After Applying Otsu's Thresholding

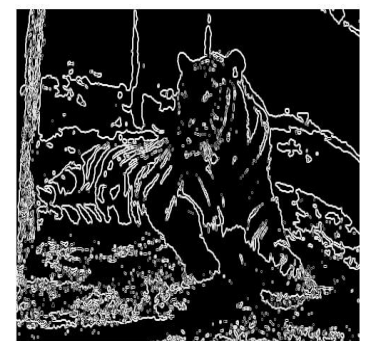

Figure 8(vii).After Applying Sobel Filter

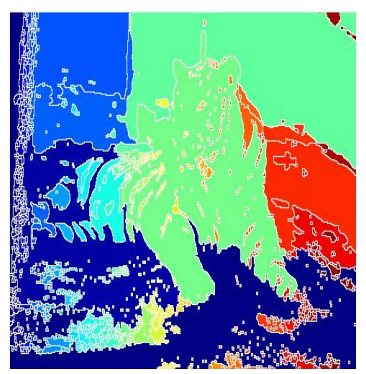

Figure 8(viii).Final Segmented Image after Applying Watershed Algorithm 
Signal \& Image Processing : An International Journal (SIPIJ) Vol.6, No.5, October 2015

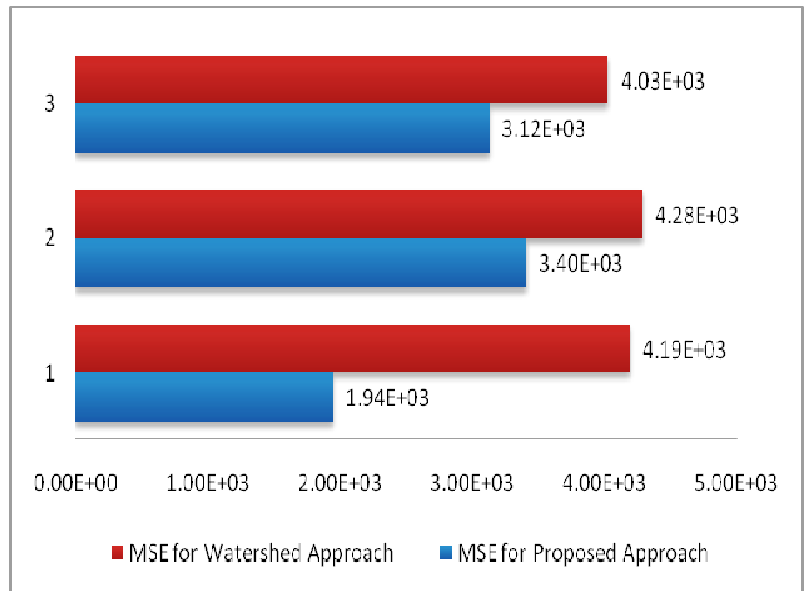

Figure 9(i).MSE Comparison for the Image 3

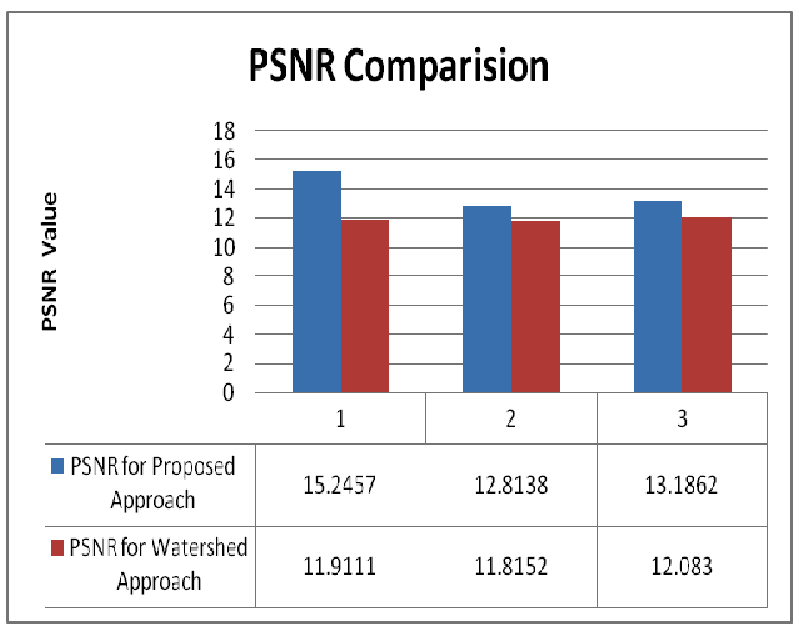

Figure 9(ii).PSNR Comparison for the Image 3

\section{Experiments for Image 4:}

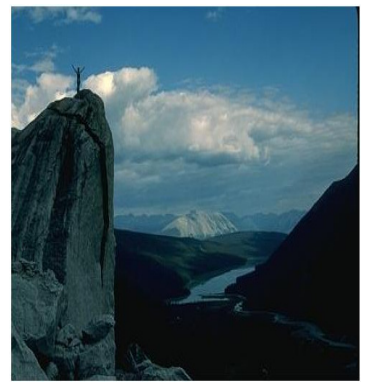

Figure 10(i).Original Image (Image 4) 
Signal \& Image Processing : An International Journal (SIPIJ) Vol.6, No.5, October 2015

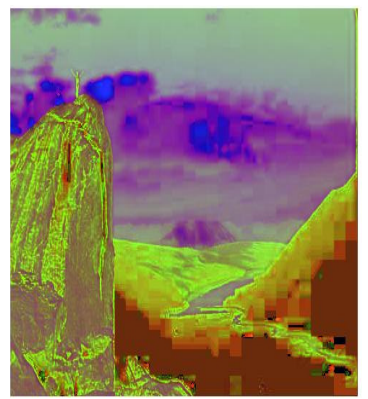

Figure 10(ii).HSV Converted Image

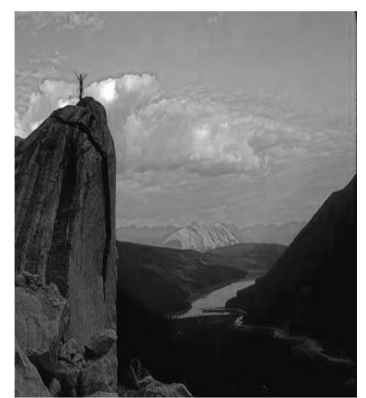

Figure 10(iii).V Channel

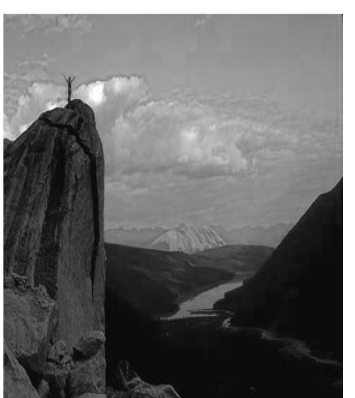

Figure 10(iv).Normalized V Channel

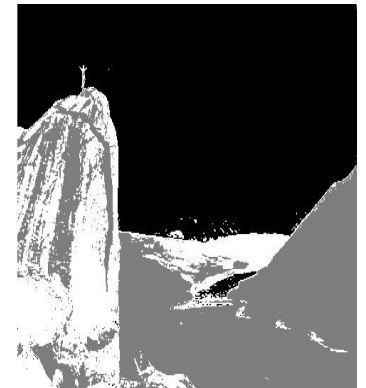

Figure 10(v).Initial Segmentation by FCM 
Signal \& Image Processing : An International Journal (SIPIJ) Vol.6, No.5, October 2015

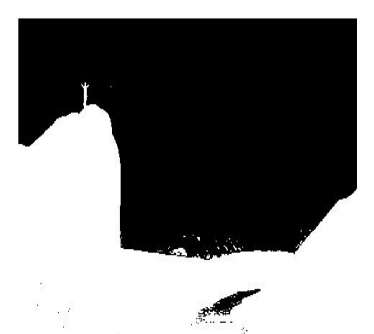

Figure 10(vi).After Applying Otsu's Thresholding

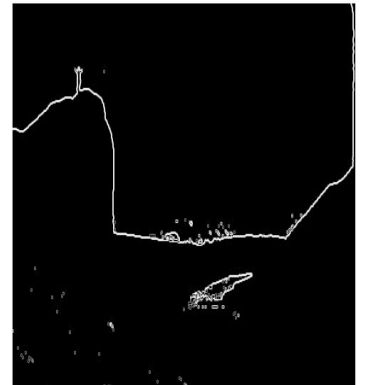

Figure 10(vii).After Applying Sobel Filter

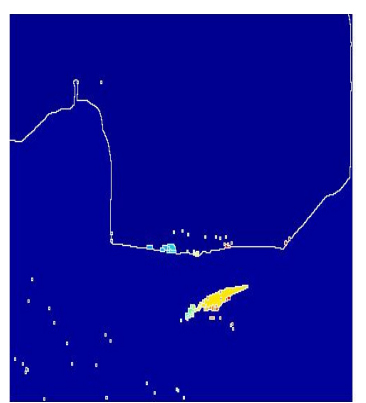

Figure 10(viii).Final Segmented Image after Applying Watershed Algorithm

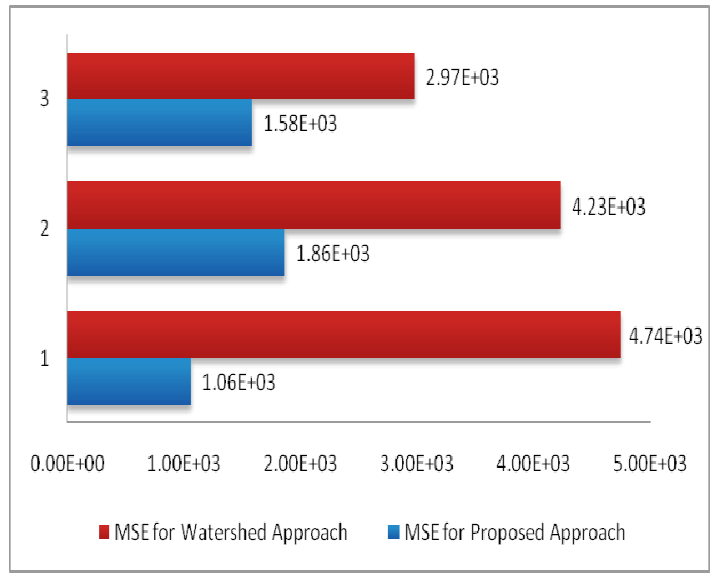

Figure 11(i).MSE Comparison for the Image 4 
Signal \& Image Processing : An International Journal (SIPIJ) Vol.6, No.5, October 2015

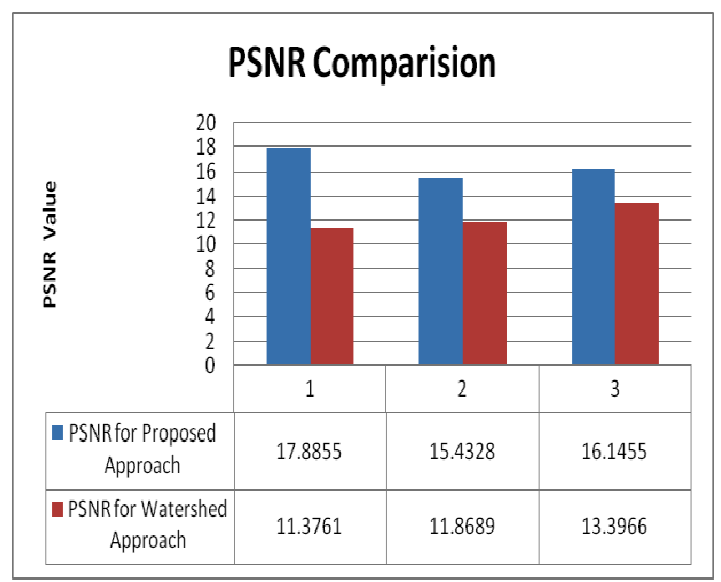

Figure 11(ii).PSNR Comparison for the Image 4

The segmentation results of the proposed approach are free from 'over segmentation' problem that generally occurs in case of watershed approach. A low MSE value and a high PSNR value always signify good image segmentation. By observing the results obtained from the experiments on all the images, it is found that our proposed approach for color image segmentation shows a lower MSE value and higher PSNR value than that of watershed approach. So, the proposed approach succeeds to produce a better segmentation result.

\section{CONCLUSION \& FUTURE ENHANCEMENT}

Today's image segmentation research gives importance on color image segmentation because of the latter's capability to deal with enhancing the image analysis process in a very fine way. Also humans' eyes have tendency to recognize an object in color form more accurately than the same in gray one. But, as color image segmentation is an emerging topic in color image analysis research so there is a requirement of an optimal technique for the same. In this paper we proposed an efficient fuzzy based watershed approach for color image segmentation task. Here the segmentation power of $\mathrm{fcm}$ is combined with the region based classical watershed algorithm. The segmentation process is done on HSV color space by extracting the $\mathrm{V}$ channel of the HSV converted image. Also, preprocessing of the input image to be segmented is given importance here. For that normalization of the extracted $\mathrm{V}$ channel is done with respect to values between 0 and 1. Again after initial segmentation by fcm, we further refined the segmented image by Otsu's global thresholding and sobel filter. Finally, watershed algorithm produces the output segmented image of the original color image. The result obtained is quite satisfactory after analyzing the MSE and PSNR values. It also overcomes the common "over segmentation" problem of watershed algorithm. In our future research, we will try to improve the performance of the proposed approach further by critically examining the pre processing and post processing issues that may further be considered with the same.

\section{REFERENCES}

[1] Chris Solomon, Toby Breckon, "Fundamentals of Digital Image Processing: A Practical Approach with Examples in Matlab", Wiley-Blackwell, ISBN: 978-0-470-84472-4.

[2] B. Georgescu, I. Shimshoni, P. Meer, "Mean Shift Based Clustering in High Dimensions: A Texture Classification Example", Intl Conf on Computer Vision, 2003. 
Signal \& Image Processing : An International Journal (SIPIJ) Vol.6, No.5, October 2015

[3] Amanpreet Kaur Bhogal, Neeru Singla,Maninder Kaur, "Color image segmentation using k-means clustering algorithm", International Journal on Emerging Technologies 1(2), 2010,pp. 18-20.

[4] B.Sathya , R.Manavalan "Image Segmentation by Clustering Methods: Performance Analysis", International Journal of Computer Applications (0975 - 8887), Volume 29- No.11, September 2011,pp 2732.

[5] D.J. Bora, A.K. Gupta, "Clustering Approach Towards Image Segmentation: An Analytical Study", IJRCAR,Vol2,Issue7,July 2014, pp. 115-124.

[6] D.J. Bora, A.K. Gupta,F A Khan, " Comparing the Performance of L*A*B* and HSV Color Spaces with Respect to Color Image Segmentation", International Journal of Emerging Technology and Advanced Engineering, Volume 5, Issue 2, February 2015, pp.192-203.

[7] Juraj Horvath, "Image Segmentation using Fuzzy C-means", SAMI 2006.

[8] Le Capitaine H, Frélicot C, "A fast fuzzy c-means algorithm for color image segmentation", In EUSFLAT-LFA 2011, Aix-les-Bains, France, 18-22 July 2011. Paris: Atlantis; 2011, pp. 1074-1081.

[9] C Mythili, V.kavitha, "Color Image Segmentation using ERKFCM", International Journal of Computer Applications 41(20), March 2012, pp.21-28.

[10] Thodeti Srikanth, Prof P.Pradeep Kumar, Ashwin Kumar, "Color Image Segmentation using Watershed Algorithm", (IJCSIT) International Journal of Computer Science and Information Technologies, Vol. 2 (5) , 2011, pp. 2332-2334.

[11] X. Zhang, Y. Shan, W. Wei, Z. Zhu, "An Image Segmentation Method Based on Improved Watershed Algorithm," Computational and Information Sciences, 2010, pp. 258-261.

[12] D.J. Bora, A.K. Gupta, "A New Approach towards Clustering based Color Image Segmentation", International Journal of Computer Applications 107(12), December 2014,pp. 23-30.

[13] A. Suphalakshmi, S. Narendran, P. Anandhakumar,"An improved fast watershed algorithm for image segmentation", Int. J. of Computational Vision and Robotics, 2010 Vol.1, No.3, pp.251 - 260.

[14] Rahman, M.H.; Islam, M.R., "Segmentation of color image using adaptive thresholding and masking with watershed algorithm," Informatics, Electronics \& Vision (ICIEV), 2013 International Conference on , vol., no., pp.1,6, 17-18 May 2013

[15] Lamia Jaafar Belaid, Walid Mourou, "Image Segmentation: A Watershed Transformation Algorithm", Image Anal Stereol, 2009;28, pp.93-102.

[16] File:HSV color solid cylinder, http://commons.wikimedia.org/wiki/File\%3AHSV_color_solid_cylinder_alpha_lowgamma.png

[17] S. Sural, G. Qian, and S. Pramanik, "Segmentation and histogram generation using the HSV color space for image retrieval," presented at IEEE International Conference on Image Processing, Rochester, New York, 2002.

[18] Shalabi, L.A., Z. Shaaban and B. Kasasbeh, "Data Mining: A Preprocessing Engine”, J. Comput. Sci., 2: 2006 , pp. 735-739.

[19] Rafael C. González, Richard Eugene Woods (2007). Digital Image Processing. Prentice Hall. ISBN 0-13168728-X, pp. 85

[20] Normalization (image processing), https://en.wikipedia.org/wiki/Normalization_(image_processing)

[21] E. Bart, S. Ullman, "Image normalization by mutual information”, in Proc. BMVC, 2004, pp. 327-336.

[22] J. C. Bezdek, "Pattern Recognition with Fuzzy Objective Function Algorithms", Plenum Press, New York, 1981.

[23] Keh-Shih Chuang, Hong-Long Tzeng, Sharon Chen, Jay Wu, Tzong-Jer Chen, 2006 : "Fuzzy c means clustering with spatial information for image segmentation", Computerized Medical Imaging and Graphics, 30, pp. $9-15$.

[24] D.J. Bora, A.K.Gupta, "Impact of Exponent Parameter Value for the Partition Matrix on the Performance of Fuzzy C Means Algorithm", arXiv preprint arXiv:1406.4007

[25] N. Otsu, "A Threshold Selection Method from Gray-Level Histograms," IEEE Transactions on Systems, Man, and Cybernetics, vol. 9, no. 1,1979, pp. 62-66.

[26] Liu Jian-zhuang, Li Wen-qing, "The Automatic threshold of gray level pictures via Two-dimentional Otsu Method", Acta Automatic Sinica, 1993

[27] Irwin Sobel, 2014, History and Definition of the Sobel Operator

[28] Raman Maini, Dr. Himanshu Aggarwal, "Study and Comparison of Various Image Edge Detection Techniques ,"International Journal of Image Processing (IJIP), Jan-Feb 2009, Volume (3) Issue (1), pp.111. 
Signal \& Image Processing : An International Journal (SIPIJ) Vol.6, No.5, October 2015

[29] S. A. Salem, N. V. Kalyankar and S. D. Khamitkar, "Image Segmentation By Using Edge Detection", (IJCSE) International Journal On Computer Science And Engineering, vol. 2, no. 3, 2010,pp. 804-807.

[30] Manisha Bhagwat, R. K. Krishna and Vivek Pise, "Simplified Watershed Transformation", International Journal of Computer Science and Communication, Vol. 1, No. 1., 2010,pp. 175-177.

[31] MATLAB Notes, http://www.mathworks.de/company/newsletters/news_notes/win02/watershed.html

[32] Meyer, Fernand, "Topographic distance and watershed lines," Signal Processing, Vol. 38, July 1994, pp. 113-125.

[33] MATLAB Demo Images, http://www.mathworks.in/products/image

[34] Berkeley Segmentation Dataset: Images, http://www.eecs.berkeley.edu/Research/Projects/CS/vision/bsds/BSDS300/html/dataset/images.html

[35] Q. Huynh-Thu, M. Ghanbari, "Scope of validity of PSNR in image/video quality assessment," Electronics Letters, vol. 44, no. 13,June 2008, pp. 800-801.

[36] T. Veldhuizen. "Measures of image quality," 2010, http://homepages.inf.ed.ac.uk/rbf/CVonline/LOCAL_COPIES/VELDHUIZEN/node18.html

\section{AUTHORS}

Mr. Dibya Jyoti Bora:

$\mathrm{PhD}$ in Computer Science pursuing (B.U. Bhopal), M.Sc. in Information Technology (University 1st Rank Holder, B.U. Bhopal), B.Sc. Honors in Mathematics (University Distinction Holder, Dibrugarh University, Assam), GATE CS/IT qualified, GSET Computer Science qualified, currently teaching P.G. students of CS and IT in the Department of Computer Science \& Applications, Barkatullah University, Bhopal. Research interests are Cluster Analysis, Image Processing ,Machine Learning and Pattern Recognition.

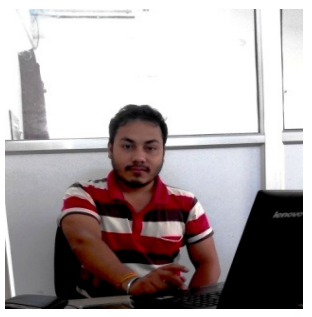

Dr. Anil Kumar Gupta:

$\mathrm{PhD}$ in Computer Science (Barkatullah University, Bhopal), HOD of the Department of Computer Science \& Applications, Barkatullah University, Bhopal. Research interests are Data Mining, Artificial Intelligence and Machine Learning.

Fayaz Ahmad Khan:

$\mathrm{PhD}$ in Computer Science pursuing (Barkatullah University, Bhopal). Research interests include Cluster Analysis, Software Engineering and Pattern Recognition. Published several research papers in international journals and conferences. He is actively involved in some emerging research problems like "Test Suite Minimization for statement Coverage testing" as one of them.
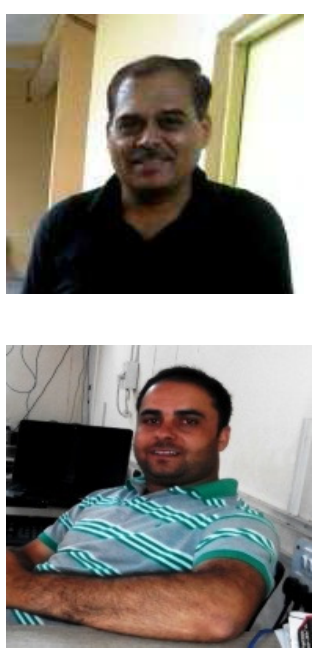